\title{
IL-22 inactivates hepatic stellate cells via downregulation of the TGF-ß1/Notch signaling pathway
}

\author{
ENRAN CHEN* ${ }^{*}$ YU CEN* ${ }^{*}$ DONGHONG LU, WEI LUO and HAIXING JIANG \\ Department of Gastroenterology, The First Affiliated Hospital of \\ Guangxi Medical University, Nanning, Guangxi 530021, P.R. China \\ Received September 20, 2017; Accepted January 16, 2018
}

DOI: $10.3892 / \mathrm{mmr} .2018 .8516$

\begin{abstract}
Interleukin-22 (IL-22) inhibits liver fibrosis by inducing hepatic stellate cell (HSC) senescence, primarily through the activation of signal transducer and activator of transcription 3 signaling. However, whether other signaling pathways are involved remains unknown. The present study assessed the regulatory mechanism between IL-22 and the Notch signaling pathway in vitro. The results revealed that IL-22 had anti-proliferative effects on HSC-T6 cells, and cellular inactivation was reflected by simultaneous inhibition of $\alpha$-smooth muscle actin, transforming growth factor- $\beta 1$ (TGF- $\beta 1$ ), tumor necrosis factor- $\alpha$ and intercellular adhesion molecule 1 . Treatment with TGF- $\beta 1$ resulted in significant Notch3 upregulation and activation of its downstream effectors Hes family basic helix-loop-helix (bHLH) transcription factor (Hes)-1, Hes-5 and Hes related family BHLH transcription factor with YRPW motif 1. Furthermore, this effect was markedly reversed by further treatment with IL-22, indicating there may be regulatory cascades of IL-22/TGF- $\beta 1$ /Notch signaling in HSC-T6 cells. The results of the present study demonstrated an inhibitory function of IL-22 towards Notch signaling in hepatic cells, providing evidence that Notch may serve as a novel target for liver fibrosis.
\end{abstract}

Correspondence to: Dr Haixing Jiang, Department of Gastroenterology, The First Affiliated Hospital of Guangxi Medical University, 6 Shuangyong Road, Nanning, Guangxi 530021, P.R. China

E-mail: gxjianghx@163.com

*Contributed equally

Abbreviations: HSC, hepatic stellate cell; $\alpha$-SMA, $\alpha$-smooth muscle actin; TGF- $\beta 1$, transforming growth factor- $\beta 1$; TNF- $\alpha$, tumor necrosis factor- $\alpha$; ICAM-1, intercellular adhesion molecule-1; HF, hepatic fibrosis; DMEM, Dulbecco's modified Eagle's medium; SDS-PAGE, sodium dodecyl sulfate-polyacrylamide gel electrophoresis; ECL, electrochemiluminescence; SD, standard deviation; ANOVA, analysis of variance

Key words: hepatic stellate cells, interleukin-22, TGF- $\beta 1$, Notch pathway

\section{Introduction}

Hepatic fibrosis (HF) is a leading cause of varices, ascites, and liver failure, and results in death in millions of patients (1). HF is usually associated with chronic liver diseases caused by infection, drugs, metabolic disorders, or autoimmune ailments (2). Liver fibrosis is characterized by the activation and proliferation of hepatic stellate cells (HSCs) (3). Tremendous progress has been achieved regarding the roles of inflammatory cells, growth factors, cytokines, and chemokines in the control of HSC activation during liver fibrogenesis $(4,5)$; however, the underlying mechanism remains unclear and needs further investigation.

It is well-established that interleukin (IL)-22 has hepatoprotective and antifibrotic functions in acute liver injury models. IL-22 is a member of the IL-10 cytokine family, and is produced primarily by Th22, Th17, and Th1 cells $(6,7)$. IL-22 effects epithelial cells, hepatocytes, and pancreatic cells, and induces innate immune responses as well as tissue protection and repair (8). In the liver, IL-22 protects the tissues from damage, and mediates tissue repair through multiple mechanisms in hepatocytes $(9,10)$. Accumulating evidence suggests that IL-22 induces HSC senescence through crosstalk with other signaling pathways such as JAK/STAT3, SOCS3 and p53 pathways, thereby inhibiting liver fibrosis (11).

The Notch pathway represents a highly conserved signaling network with essential roles in the regulation of key cellular processes and functions, many of which are critical for development (12-14). Furthermore, emerging evidence indicates that it is also essential for fibrosis, thus affecting the pathogenesis of chronic fibro proliferative diseases in diverse organs and tissues $(15,16)$. Bansal et al $(16)$ demonstrated the functional effects of Notch signaling on HSC activation and M1/M2 polarization of macrophages in liver fibrosis, although the molecular mechanism remains elusive. Although the Notch signaling pathway is involved in human fibrotic diseases affecting the lung, kidney, and peritoneum $(12,13)$, the interaction between IL-22 and Notch signaling in HSCs remains elusive. The evidence currently available, suggests that IL-22 may downregulate Notch3 (17-19); therefore, we concentrated on this member of the Notch family. In this study, we confirmed that IL-22 inhibited HSC activation through TGF- $\beta$-mediated downregulation of the Notch pathway. Therefore, selective 
inhibition of Notch signaling may be a novel anti-fibrotic strategy for liver fibrosis treatment.

\section{Materials and methods}

Cell lines and reagents. HSC-T6 cells were purchased from the Chinese Academy of Sciences (Beijing, China), and cultured in Dulbecco's modified Eagle's medium (DMEM) (Gibco; Thermo Fisher Scientific, Inc., Waltham, MA, USA) supplemented with $10 \%$ fetal bovine serum (FBS; Gemini, Australia). Cells were maintained at $37^{\circ} \mathrm{C}$ in a humidified atmosphere containing $5 \% \mathrm{CO}_{2}$. IL-22 was purchased from R\&D Systems (Minneapolis, MN, USA) and TGF- $\beta 1$ from Cusabio Biotech Co., Ltd. (Wuhan, China).

Cell viability assay. The effects of IL-22 on HSC-T6 cell viability were evaluated with the Cell Counting Kit-8 (CCK-8) (Dojindo Molecular Technologies, Inc., Kumamoto, Japan) cell proliferation assay. Cells were seeded in 96-well plates at a density of $1 \times 10^{4}$ cells/well. After overnight growth, the cells were incubated with various concentrations of IL-22 (0-1,000 pg/ml) (R\&D Systems). After cell treatment for $24 \mathrm{~h}$, $10 \mu \mathrm{l} \mathrm{CCK}-8$ solution was added into each well. Absorbance was measured at $450 \mathrm{~nm}$ with background at $655 \mathrm{~nm}$, using a micro plate reader (Bio-Rad Laboratories, Hercules, CA, USA). All experiments were repeated three times.

Cell apoptosis assay. Apoptosis was assessed, after $24 \mathrm{~h}$ of treatment with IL-22, with the APC Annexin V Apoptosis Detection kit and PE Active Caspase-3 Apoptosis kit (both from BD Pharmingen, San Diego, CA, USA) according to the manufacturers' instructions. Briefly, HSC-T6 cells were treated with IL-22, followed by staining in fluorescence activated cell sorter (FACS) buffer (PBS, 2\% bovine serum albumin, $0.1 \%$ sodium azide) for $10 \mathrm{~min}$ at $4^{\circ} \mathrm{C}$. Finally, cells were washed and assessed on BD FACSCalibur Flow Cytometer (BD Pharmingen). Data were analyzed with the FlowJo software (Tree Star, Ashland, OR, USA).

Reverse transcription-quantitative polymerase chain reaction (RT-qPCR). RNA was extracted from cells using TRIzol (Invitrogen Life Technologies, Carlsbad, CA, USA) according to the manufacturer's instructions. Reverse transcription was performed with a cDNA Reverse Transcription kit (Takara Bio, Inc., Otsu, Japan). RT-qPCR was performed with a SYBR-Green Master Mix kit (Takara Bio, Inc.). Relative mRNA levels were normalized to GAPDH mRNA expression, and calculated by the $2^{-\triangle \Delta C q}$ method. The primer sequences are summarized in Table I.

Immunoblot. Samples were homogenized in lysis buffer containing Tris- $\mathrm{HCl}, \mathrm{NP}-40, \mathrm{NaCl}$, ethylene diamine-tetra acetic acid, $\mathrm{NaN}_{3}$, phenylmethylsulfonyl fluoride, aprotinin,

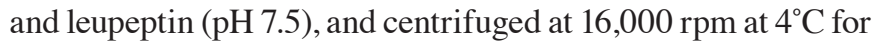
$10 \mathrm{~min}$. Protein concentration was determined by BCA (Pierce Biotechnology, Inc., Rockford, IL, USA). Equal amounts of total protein $(30 \mu \mathrm{g})$ were separated by $10-15 \%$ sodium dodecyl sulfate-polyacrylamide gel electrophoresis (SDS-PAGE) and transferred onto PVDF membranes. Antibodies against SMA were obtained from Abcam (Cambridge, MA, USA).
Anti-GAPDH was from Santa Cruz Biotechnology, Inc. (Dallas, TX, USA). Anti-Notch3 was manufactured by Abcam (Cambridge, UK). Then membranes were incubated with various primary antibodies overnight at $4^{\circ} \mathrm{C}$, followed by incubation with secondary antibodies and detection by enhanced chemiluminescence (ECL) (Odyssey; LI-COR Biosciences, Lincoln, NE, USA).

Statistical analysis. Data are mean \pm standard deviation (SD) from three independent experiments. Statistical analysis was performed by unpaired Student's t-test, with two tailed $\mathrm{P}<0.05$ considered statistically significant. Multiple groups were assessed by one-way analysis of variance and Dunnett's post hoc test. The SPSS 16.0 software (SPSS Inc., Chicago, IL, USA) was used for statistical analysis.

\section{Results}

IL-22 treatment inhibits HSC-T6 cells proliferation without inducing apoptosis. We first assessed the anti-proliferative effects of IL-22 on hepatic cells. Increasing concentrations of IL-22 were respectively applied to HSC-T6 cells, and cell proliferation was determined by CCK-8 assay. Cell viability was significantly decreased at high IL-22 amounts (Fig. 1A). However, IL-22 had no effect on apoptosis inHSC-T6 cells. As shown in Fig. 1B and C, there was no significant difference between IL-22 treatment and control groups. Taken together, these results indicated that IL-22 may exert anti-proliferative effects, independent of apoptosis.

IL-22 downregulates $\alpha$-SMA and proinflammatory cytokines in HSC-T6 cells. Increased expression levels of $\alpha$-SMA and collagen are commonly recognized as biomarkers of HSC activation (3). Next, we assessed the expression levels of $\alpha$-SMA and multiple hepatocyte-associated proinflammatory cytokines to verify the inhibitory effects of IL-22 on HSC-T6 proliferation. Interestingly, application of IL-22 inhibited $\alpha$-SMA expression in a dose-dependent manner (Fig. 2A). Furthermore, we assessed whether common pro-inflammatory cascades were inhibited by IL-22. TGF- $\beta$ and tumor necrosis factor- $\alpha$ (TNF- $\alpha$ ) are the most important mediators of inflammatory responses in HSCs, while ICAM-1 is an essential cell adhesion molecule $(8,9)$. As shown in Fig. 2B-E, the mRNA levels of TGF- $\beta$, TNF- $\alpha$, and ICAM-1 were simultaneously decreased by IL-22 treatment in HSC-T6 cells, suggesting that these effectors are downstream of IL-22. Collectively, HSC inactivation by IL-22 was confirmed by the downregulation of $\alpha$-SMA and related-inflammatory cytokines.

TGF- $\beta 1$ activates the Notch pathway in HSC-T6 cells. We further explored the possible downstream effectors upon TGF- $\beta$ stimulation. Several studies previously described a functional interaction between the TGF- $\beta$ superfamily of proteins and Notch signaling in multiple biological processes $(15,16)$. The results demonstrated that Notch3 levels were remarkably increased in HSC-T6 cells after TGF- $\beta 1$ treatment, in a dose-dependent manner (Fig. 3A), in line with densitometry analysis (Fig. 3B). Furthermore, not only was the expression of Notch3 receptor increased by TGF- $\beta 1$, but Notch pathway activation was confirmed, as indicated by substantial upregulation 
Table I. Primers used for reverse transcription-quantitative polymerase chain reaction.

\begin{tabular}{|c|c|c|}
\hline Gene & Primer $\left(5^{\prime}-3^{\prime}\right)$ & Base pairs (bp) \\
\hline GAPDH & $\begin{array}{l}\text { Forward: CAGCTTTTGAAGGGGAACGC } \\
\text { Reverse: TCATGCTCAGAAGTGGCTGG }\end{array}$ & 182 \\
\hline Hes-1 & $\begin{array}{l}\text { Forward: TCAACACGACACCGGACAA } \\
\text { Reverse: GTGCTTCACTGTCATTTCCAGA }\end{array}$ & 120 \\
\hline Hes-5 & $\begin{array}{l}\text { Forward: AGCCGGTGGTGGAGAAGAT } \\
\text { Reverse: AGTTTGGAGTTGGGCTGGTG }\end{array}$ & 100 \\
\hline Неy-1 & $\begin{array}{l}\text { Forward: AGCTGAGATCTTGCAGATGACTGTG } \\
\text { Reverse: AGCCAGGCATTCCCGAAAC }\end{array}$ & 109 \\
\hline TGF- $\beta$ & $\begin{array}{l}\text { Forward: ATTCCTGGCGTTACCTTGG } \\
\text { Reverse: AGCCCTGTATTCCGTCTCCT }\end{array}$ & 120 \\
\hline TNF- $\alpha$ & $\begin{array}{l}\text { Forward: CAGGTTCCGTCCCTCTCATA } \\
\text { Reverse: TGCCAGTTCCACATCTCG }\end{array}$ & 100 \\
\hline ICAM-1 & $\begin{array}{l}\text { Forward: ATGGACGCTCACCTTTAGCA } \\
\text { Reverse: TCTCCCAGGCATTCTCTTTG }\end{array}$ & 109 \\
\hline
\end{tabular}

TGF- $\beta$, transforming growth factor- $\beta$; TNF- $\alpha$, tumor necrosis factor- $\alpha$; ICAM- 1 , intercellular adhesion molecule- 1 ; Hes- 1 , Hes family basic helix-loop-helix transcription factor-1; Hey-1, Hes related family basic helix-loop-helix transcription factor with YRPW motif 1; IL, interleukin .
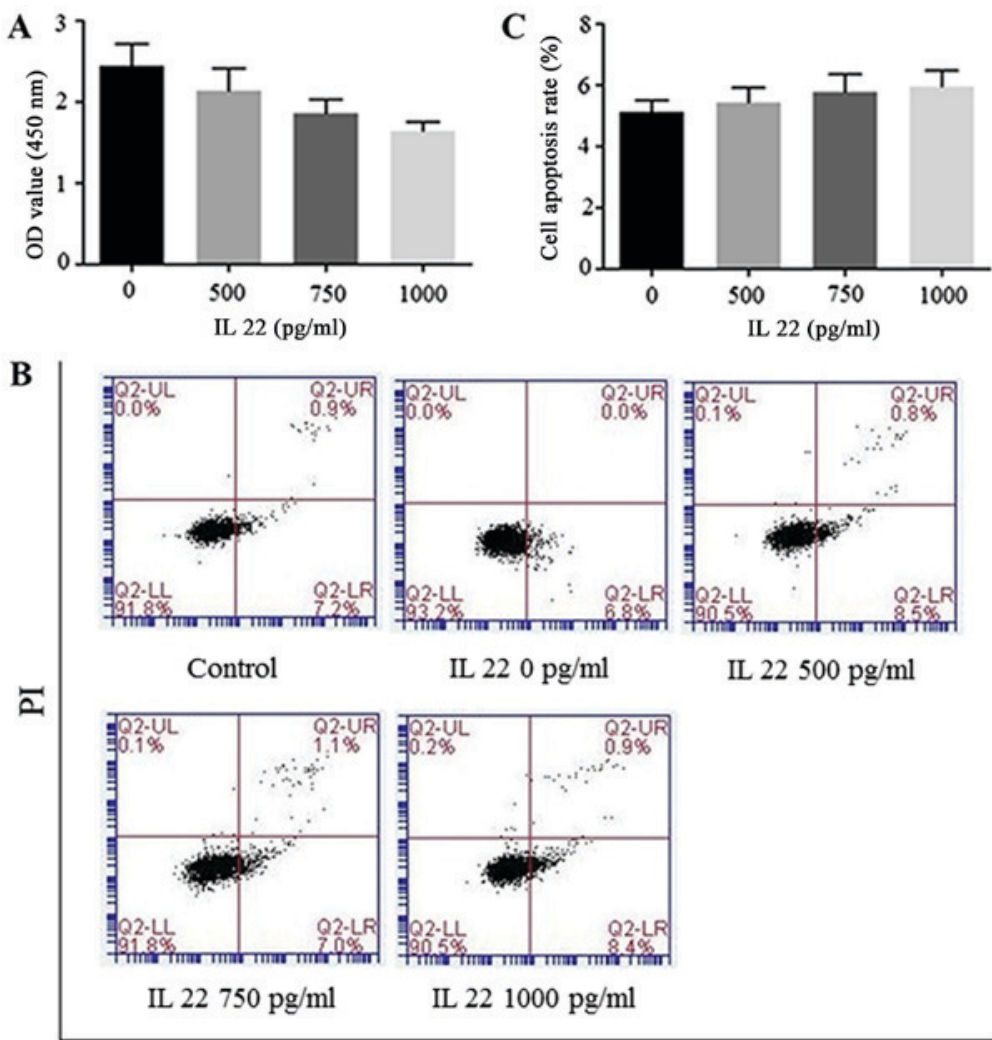

Annexin V

Figure 1. IL-22 inhibits the proliferation of HSC-T6 cells without affecting apoptosis. (A) The effects of IL-22 on HSC-T6 cell proliferation. (B) Flow cytometric analysis of HSC-T6 cells following IL-22 treatment. (C) Apoptosis rates post IL-22 treatment in HSC-T6 cells. IL, interleukin; PI, propidium iodide; OD, optical density.

of the downstream effectors Hes family basic helix-loop-helix transcription factor-1 (Hes-1), Hes-5 and Hey-1 (Fig. 3C-E). Taken together, these results indicated that TGF- $\beta$ is responsible for the activation of Notch signaling in HSC-T6 cells.
IL-22 treatment inhibits the Notch pathway in HSC-T6 cells. As TGF- 31 -induced Notch3 upregulation, we wondered if such regulation can be altered by IL-22. Intriguingly, Notch3 expression was reduced after incubation with IL-22 (Fig. 4A and B). 

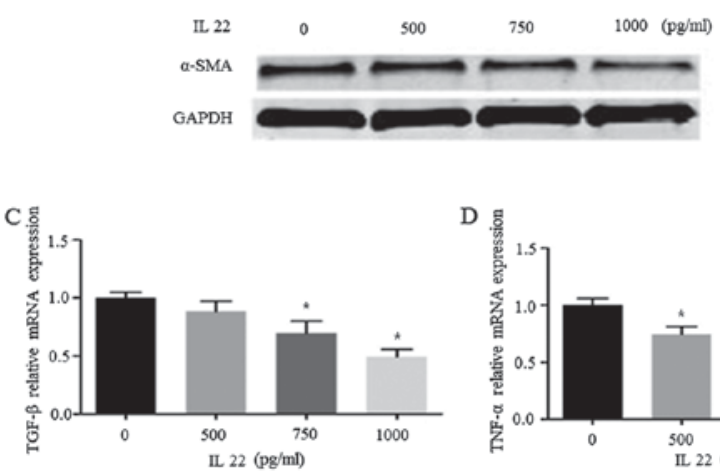

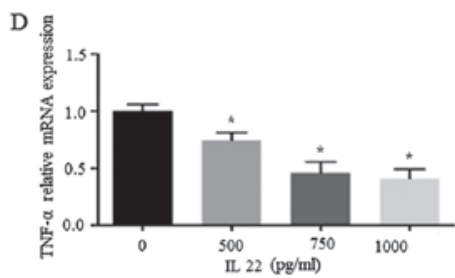

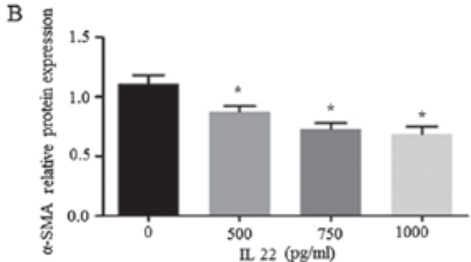

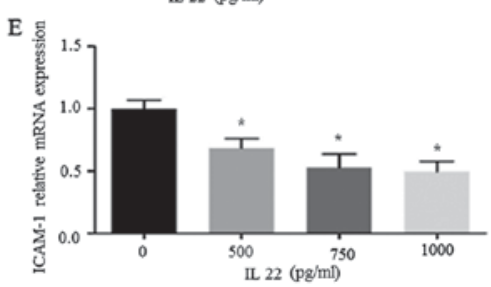

Figure 2. IL-22 decreases the expression levels of $\alpha$-SMA and proinflammatory cytokines in HSC-T6 cells. (A) Immunoblot detection of the $\alpha$-SMA protein (B) Densitometric histogram representing the percentages of $\alpha$-SMA protein expression. Gene mRNA expression levels of (C) TGF- $\beta$, (D) TNF- $\alpha$ and (E) ICAM-1 following IL-22 treatment. "P $<0.05$ vs. 0 pg/ml IL-22. SMA, smooth muscle actin; IL, interleukin; TGF- $\beta$, transforming growth factor- $\beta$; TNF- $\alpha$, tumor necrosis factor- $\alpha$; ICAM-1, intercellular adhesion molecule-1.

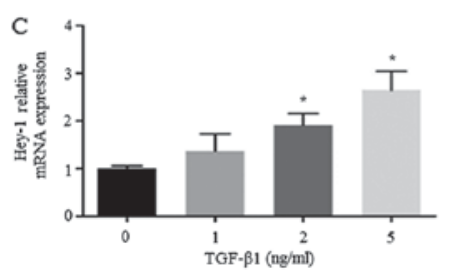

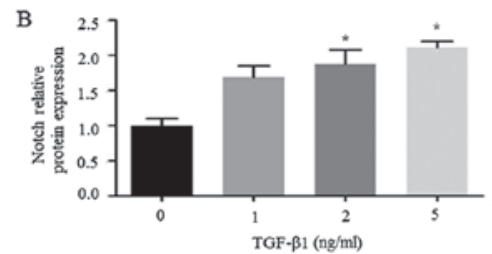

TOF- $\beta 1$ (ng/ml)

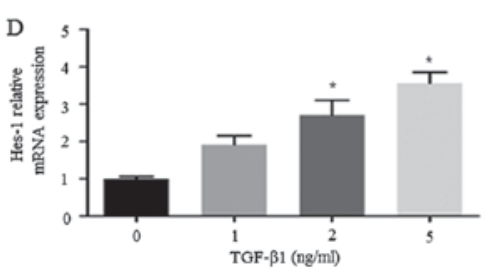

E

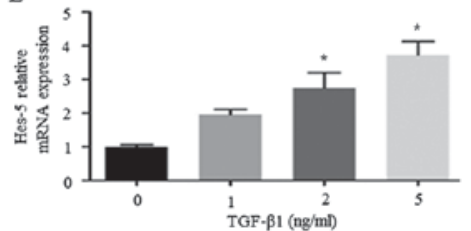

Figure 3. Notch signaling in HSC-T6 cells is activated by TGF- $\beta 1$ treatment. (A) Immunoblot detection of Notch3. (B) Relative Notch3 protein expression. (C) Hey-1, (D) Hes-1 and (E) Hes-5 mRNA expression levels following TGF- $\beta 1$ treatment. "P<0.05 vs. 0 ng/ml TGF- $\beta 1$. TGF- $\beta 1$, transforming growth factor- $\beta 1$; Hes-1, Hes family basic helix-loop-helix transcription factor-1; Hey-1, Hes related family basic helix-loop-helix transcription factor with YRPW motif 1 ; IL, interleukin.
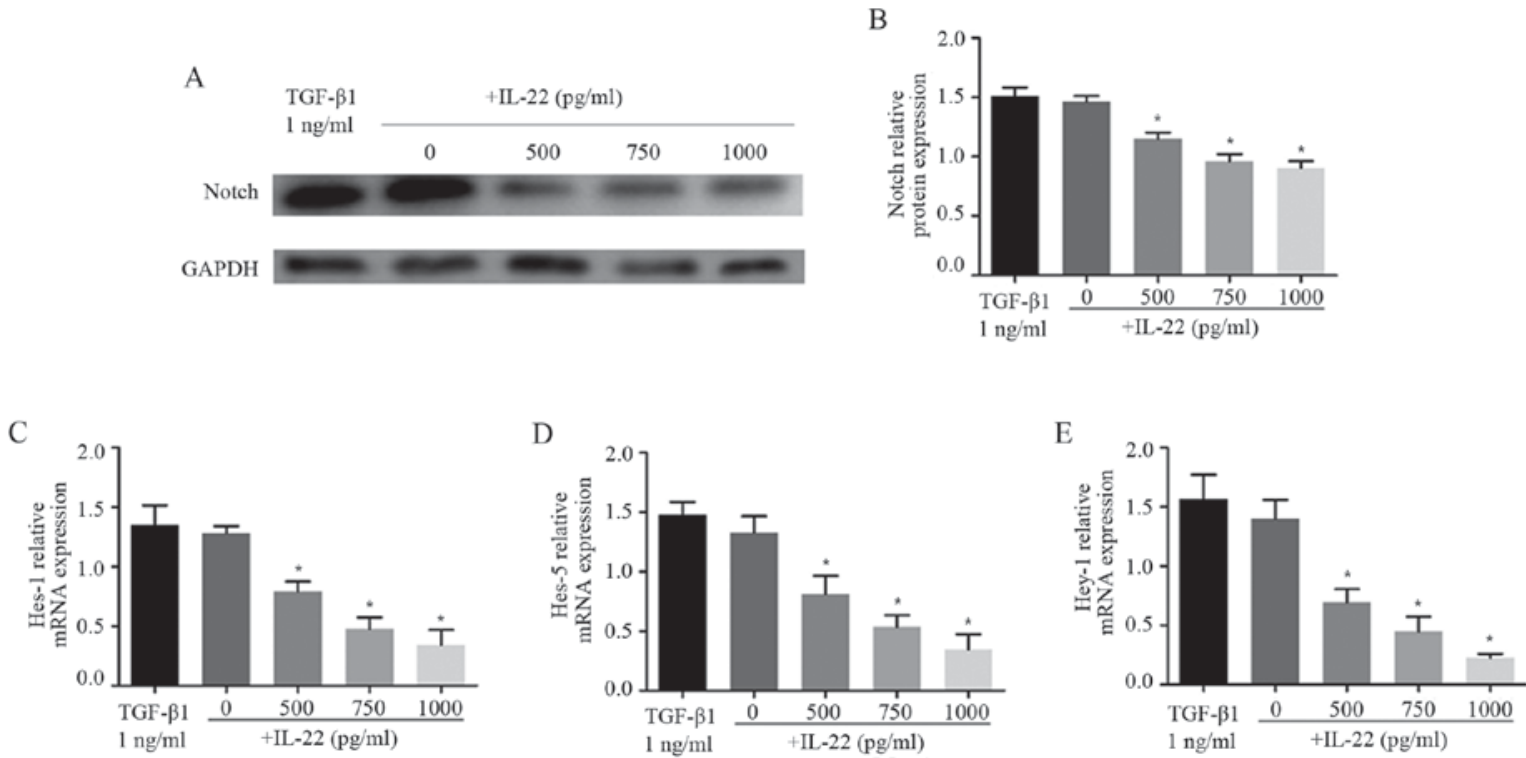

Figure 4. IL-22 inhibits Notch signaling in HSC-T6 cells. (A) Immunoblot detection of the Notch3 protein. (B) Relative Notch3 protein expression. (C) Hes-1, (D) Hes-5 and (E) Hey-1 mRNA expression levels following IL-22 treatment. ${ }^{*} \mathrm{P}<0.05$ vs. 0 pg/ml IL-22. TGF- $\beta 1$, transforming growth factor- $\beta 1$; Hes-1, Hes family basic helix-loop-helix transcription factor-1; Hey-1, Hes related family basic helix-loop-helix transcription factor with YRPW motif 1; IL, interleukin. 
Meanwhile, the effect of IL-22 on the TGF- $\beta 1$-induced Notch3 increase was evaluated. The results showed that IL-22 decreased Notch3 expression in a dose dependent way in HSC-T6 cells (Fig. 4A and B), suggesting Notch3 to be a downstream signal transducer of IL-22-related TGF- $\beta 1$ inhibition. These results further confirmed that both Notch3 expression and pathway activation were impaired by IL-22. Compared with the TGF- $\beta 1$ group, combined treatment with IL-22 significantly decreased Hes-1, Hes-5 and Hey-1 mRNA levels (Fig. 4C-E). Taken together, these findings suggested that IL-22 plays a role in regulation of the Notch signaling pathway.

\section{Discussion}

The present study demonstrated that IL-22 reduces HSC-T6 activation through Notch 3 inhibition, suggesting the protective role of IL-22 in liver fibrosis in vitro and revealing a potential target for liver fibrosis.

Liver fibrosis is a chronic, but reversible wound healing response characterized by a tight interplay between inflammatory and matrix-producing cellular pathways (5). During this process, various signaling pathways are involved in the activation of HSCs. Therefore, targeting a specific signaling pathway may be helpful in identifying effective treatment tools for liver fibrosis. To date, several signaling pathways have been found to be closely related to liver fibro-genesis. Notch signaling is considered a major signaling mechanism in liver biology as well as multiple pathological conditions, from liver damage to carcinogenesis (20). In addition, Notch3 and Jagged1 (mainly related to HSC activation) are upregulated in diseased human livers as well as mouse models $(21,22)$. The activated Notch pathway is involved in some fibrotic diseases. Zhu et al (23) reported that Notch signaling is highly activated in rats with fibrotic peritoneum induced by peritoneal dialysis fluid; indeed, blocking Notch signaling significantly attenuates peritoneal fibrosis. Studies also showed that Notch signaling is involved in the activation of HSCs in vivo, with transient knockdown of Notch3 antagonizing TGF- $\beta 1$-induced expression of $\alpha$-SMA and collagen I in HSC-T6 cells (19). In the present study, TGF- $\beta 1$ was used to activate HSCs, and Notch3, TGF- $\beta$, TNF- $\alpha$, and ICAM-1 expression levels were significantly increased, indicating that the Notch pathway is involved in TGF- $\beta 1$ induced activation of HSCs.

IL-22 inhibits HSCs through the signal transducer and activator of transcription 3 (STAT3) signaling pathway $(10,24)$. Meanwhile, studies reported that Notch is regulated by TGF- $\beta 1(15,16)$; however, whether TGF- $\beta 1$ is involved in IL-22 induced inhibition of HSCs remains unclear. In the present study, TGF- $\beta 1$ significantly increased the expression levels of Notch3, Hes-1, Hes-5 and Hey-1, which were significantly rescued by IL-22. Taken together, these findings suggested that IL-22 inhibits HSC activation may through the regulation of TGF- $\beta 1 /$ Notch signaling. However, how IL-22 modulates TGF- $\beta 1 /$ Notch signaling, and whether this is directly related to the expression of $\alpha$-SMA, needs to be further investigated.

In conclusion, this study firstly revealed a biological interaction between the pro-inflammatory cytokine IL-22 and Notch signaling in preventing liver fibrosis, with TGF- $\beta 1$ required for the regulatory process.

\section{References}

1. Ginès $\mathrm{P}$, Càrdenas $\mathrm{A}$, Arroyo V and Rodès J: Management of cirrhosis and ascites. N Engl J Med 350: 1646-1654, 2004.

2. Pan CX, Tang J, Wang XY, Wu FR, Ge JF and Chen FH: Role of interleukin-22 in liver diseases. Inflamm Res 63: 519-525, 2014.

3. Puche JE, Saiman Y and Friedman SL: Hepatic stellate cells and liver fibrosis. Compr Physiol 3: 1473-1492, 2013.

4. Lee UE and Friedman SL: Mechanisms of hepatic fibrogenesis. Best Pract Res Clin Gastroenterol 25: 195-206, 2011.

5. Hernandez-Gea V and Friedman SL: Pathogenesis of liver fibrosis. Annu Rev Pathol 6: 425-456, 2011.

6. Sonnenberg GF, Fouser LA and Artis D: Border patrol: Regulation of immunity, inflammation and tissue homeostasis at barrier surfaces by IL-22. Nat Immunol 12: 383-390, 2011.

7. Zenewicz LA and Flavell RA: Recent advances in IL-22 biology. Int Immunol 23: 159-163, 2011.

8. Sertorio M, Hou X, Carmo RF, Dessein H, Cabantous S, Abdelwahed M, Romano A, Albuquerque F, Vasconcelos L, Carmo T, et al: IL-22 and IL-22 binding protein (IL-22BP) regulate fibrosis and cirrhosis in hepatitis $\mathrm{C}$ virus and schistosome infections. Hepatology 61: 1321-1331, 2015.

9. Carmo RF, Cavalcanti MS and Moura P: Role of interleukin-22 in chronic liver injury. Cytokine 98: 107-144, 2017.

10. Zhang YM, Liu ZR, Cui ZL, Yang C, Yang L, Li Y and Shen ZY: Interleukin-22 contributes to liver regeneration in mice with concanavalin A-induced hepatitis after hepatectomy. World J Gastroenterol 22: 2081-2091, 2016.

11. Kong X, Feng D, Wang H, Hong F, Bertola A, Wang FS and Gao B: Interleukin-22 induces hepatic stellate cell senescence and restricts liver fibrosis in mice. Hepatology 56: 1150-1159, 2012.

12. Bolòs V, Grego-Bessa $\mathbf{J}$ and de la Pompa JL: Notch signaling in development and cancer. Endocr Rev 28: 339-363, 2007.

13. Nichols AM, Pan Y, Herreman A, Hadland BK, De Strooper B, Kopan R and Huppert SS: Notch pathway is dispensable for adipocyte specification. Genesis 40: 40-44, 2004.

14. Liu T, Hu B, Choi YY, Chung M, Ullenbruch M, Yu H, Lowe JB and Phan SH: Notch1 signaling in FIZZ1 induction of myofibroblast differentiation. Am J Pathol 174: 1745-1755, 2009.

15. Bielesz B, Sirin Y, Si H, Niranjan T, Gruenwald A, Ahn S, Kato H, Pullman J, Gessler M, Haase VH and Susztak K: Epithelial Notch signaling regulates interstitial fibrosis development in the kidneys of mice and humans. J Clin Invest 120: 4040-4054, 2010.

16. Bansal R, van Baarlen J, Storm G and Prakash J: The interplay of the Notch signaling in hepatic stellate cells and macrophages determines the fate of liver fibrogenesis. Sci Rep 5: 18272, 2015.

17. Trehanpati N, Shrivastav S, Shivakumar B, Khosla R, Bhardwaj S, Chaturvedi J, Sukriti, Kumar B, Bose S, Mani Tripathi D, et al: Analysis of Notch and TGF- $\beta$ signaling expression in different stages of disease progression during hepatitis B virus infection. Clin Transl Gastroenterol 3: e23, 2012.

18. Lu DH, Guo XY, Qin SY, Luo W, Huang XL, Chen M, Wang JX, Ma SJ, Yang XW and Jiang HX: Interleukin-22 ameliorates liver fibrogenesis by attenuating hepatic stellate cell activation and downregulating the levels of inflammatory cytokines. World $\mathrm{J}$ Gastroenterol 21: 1531-1545, 2015.

19. Chen YX, Weng ZH and Zhang SL: Notch3 regulates the activation of hepatic stellate cells. World J Gastroenterol 18: 1397-1403, 2012.

20. Geisler F and Strazzabosco M: Emerging roles of Notch signaling in liver disease. Hepatology 61: 382-392, 2015.

21. Chen Y, Zheng S, Qi D, Zheng S, Guo J, Zhang S and Weng Z: Inhibition of Notch signaling by a $\gamma$-secretase inhibitor attenuates hepatic fibrosis in rats. PLoS One 7: e46512, 2012.

22. Wei X, Wang JP, Hao CQ, Yang XF, Wang LX, Huang CX, Bai XF, Lian JQ and Zhang Y: Notch signaling contributes to liver inflammation by regulation of interleukin-22-producing cells in hepatitis B virus infection. Front Cell Infect Microbiol 6: 132,2016

23. Zhu F, Li T, Qiu F, Fan J, Zhou Q, Ding X, Nie J and Yu X: Preventive effect of Notch signaling inhibition by a gamma-secretase inhibitor on peritoneal dialysis fluid-induced peritoneal fibrosis in rats. Am J Pathol 176: 650-659, 2010.

24. Mühl H: STAT3, a key parameter of cytokine-driven tissue protection during sterile inflammation-the case of experimental acetaminophen (Paracetamol)-induced liver damage. Front Immunol 7: 163, 2016. 